\title{
Joint Source-Channel Coding via Statistical Mechanics: Thermal Equilibrium Between the Source and the Channel *
}

\author{
Neri Merhav \\ Department of Electrical Engineering \\ Technion - Israel Institute of Technology \\ Haifa 32000, ISRAEL
}

\begin{abstract}
We examine the classical joint source-channel coding problem from the viewpoint of statistical physics and demonstrate that in the random coding regime, the posterior probability distribution of the source given the channel output is dominated by source sequences, which exhibit a behavior that is highly parallel to that of thermal equilibrium between two systems of particles that exchange energy, where one system corresponds to the source and the other corresponds to the channel. The thermodynamical entopies of the dual physical problem are analogous to conditional and unconditional Shannon entropies of the source, and so, their balance in thermal equilibrium yields a simple formula for the mutual information between the source and the channel output, that is induced by the typical code in an ensemble of joint source-channel codes under certain conditions. We also demonstrate how our results can be used in applications, like the wiretap channel, and how can it be extended to multiuser scenarios, like that of the multiple access channel.
\end{abstract}

Index Terms: joint source-channel coding, statistical physics, thermal equilibrium, mutual information, entropy.

\section{Introduction}

Consider the following two seemingly unrelated problems, which serve as simple special cases of a more general setting we study later in this paper:

The first is an elementrary problem in statistical physics: We have two subsystems of particles which are brought into thermal equilibirium with each other as well as with the environment (a

\footnotetext{
${ }^{*}$ Part of this work was carried out during a visit in Hewlett-Packard Laboratories, Palo Alto, CA, U.S.A., in the Summer of 2008.
} 
heat bath) at temperature $T$. The first subsystem consists of $N$ particles having magnetic moments (spins), $\left\{s_{i}\right\}$, each of which may be oriented either in the direction of an applied external magnetic field $B$, in which case $s_{i}=+1$, or in the opposite direction, in which case $s_{i}=-1$, and its energy in both cases is given by $-s_{i} B$ (up to a certain multiplicative constant, which carries the appropriate physical units, and which is irrelevant for the purpose of this discussion). In the second subsystem, there are $n$ non-interacting particles $\left\{s_{i}^{\prime}\right\}_{i=1}^{n}$, each one of which may lie in one of two possible states: the state $s_{i}^{\prime}=0$, in which the particle has zero energy, and the state $s_{i}^{\prime}=1$, in which it has energy $e_{0}$. What is the average energy possessed by each one of these subsystems in equilibrium, as functions of $e_{0}, T, n, N$, and $B$ ?

The second problem is in Information Theory, in particular, it is in joint source-channel coding, where some of the notation used is deliberately chosen to be the same as in the previous paragraph: A binary memoryless source generates a vector $s$ of symbols $\left(s_{1}, s_{2}, \ldots, s_{N}\right), s_{i} \in\{+1,-1\}, i=$ $1, \ldots, N$, with probabilities $q=\operatorname{Pr}\left\{S_{i}=+1\right\}$ and $1-q=\operatorname{Pr}\left\{S_{i}=-1\right\}$. This vector is encoded into a binary channel codeword $\boldsymbol{x}(\boldsymbol{s})$ of length $n$ and transmitted over a binary symmetric channel (BSC) with a crossover probability $p<1 / 2$, and a binary $n$-vector $\boldsymbol{y}$ is received at the channel output. Consider the posterior distribution

$$
P(\boldsymbol{s} \mid \boldsymbol{y})=\frac{P(\boldsymbol{s}) W(\boldsymbol{y} \mid \boldsymbol{x}(\boldsymbol{s}))}{\sum_{\boldsymbol{s}^{\prime}} P\left(\boldsymbol{s}^{\prime}\right) W\left(\boldsymbol{y} \mid \boldsymbol{x}\left(\boldsymbol{s}^{\prime}\right)\right)}
$$

where $P(\boldsymbol{s})$ and $W(\boldsymbol{y} \mid \boldsymbol{x})$ are the probability distributions that govern the source and the channel, respectively, as described above. Thus, clearly, $P(\boldsymbol{s} \mid \boldsymbol{y})$ is proportional to $P(\boldsymbol{s}) W(\boldsymbol{y} \mid \boldsymbol{x}(\boldsymbol{s}))$, or equivalently, $\ln P(\boldsymbol{s} \mid \boldsymbol{y})$ is (within a term that is independent of $\boldsymbol{s}$ ) given by $\ln P(\boldsymbol{s})+\ln W(\boldsymbol{y} \mid \boldsymbol{x}(\boldsymbol{s})$ ). For a typical code drawn uniformly at random from the ensemble of codes, what are the relative contributions of the source and the channel to this quantity, for those vectors $s$ that dominate $P(\boldsymbol{s} \mid \boldsymbol{y})$ (i.e., those that capture the vast majority of the posterior probability)?

It turns out, as we shall see in Section 3 below, that the two problems have virtually identical answers (in a sense that will be made clear and precise therein), provided that the parameters $T$ and $B$ of the first problem are related to the parameters $p$ and $q$ of the second problem by

$$
p=\frac{\exp \left\{-e_{0} / k T\right\}}{1+\exp \left\{-e_{0} / k T\right\}}
$$


and

$$
q=\frac{\exp \{B / k T\}}{2 \cosh (B / k T)}
$$

or equivalently,

$$
e_{0}=k T \ln \frac{1-p}{p}
$$

and

$$
B=\frac{k T}{2} \ln \frac{q}{1-q}
$$

where $k$ is Boltzmann's constant.

Thermal equilibrium between the two subsystems in the above described physical problem, dictates a certain balance between their thermodynamical entropies in order to arrive at the maximum total entropy (by the second law of thermodynamics) for the total energy possessed by the entire system at the given temperature $T$. As the thermodynamical entropy, in its statistical-mechanical definition, is intimately related to the Shannon entropy, it turns out that this equilibrium relation between the thermodynamical entropies of the physical problem, gives rise to an analogous relation between Shannon entropies pertaining to the joint source-channel coding problem in the random coding regime. In particular, it relates the entropy of the source to its conditional entropy given the channel output, whose difference is exactly the mutual information between the source and the channel output. The final outcome of this is a simple formula for calculating the mutual information rate between the input and the output of a coded system for the typical code in a given ensemble under certain conditions. This calculation builds strongly on the random energy model (REM) of spin glasses due to Derrida $[3,4,5]$ and its relation to the random code ensemble (RCE) as described in [12].

Clearly, under the regime of reliable communication, the mutual information rate between the source and the channel output coincides with the entropy rate of the source, as the conditional entropy rate of the source given the channel output vanishes. Thus, the problem of calculating the mutual information under reliable communication conditions is easy and in fact, not quite interesting. The same calculation, however, when the conditions of reliable communication are not met, appears less trivial. But what would be the motivation for such a calculation?

Here are just a few examples that motivate this: Consider a user that, in addition to its desired signal, receives also a relatively strong interfering signal (codeword), which is intended to 
other users, and which comes from a codebook whose rate exceeds the capacity of this crosstalk channel between the interferer and our user, so that the user cannot fully decode this interference. Nonetheless, our user would like to learn as much as possible on the interfering signal for many possible reasons: For example, the user would like to learn the interference signal in order to identify where it originates from, or in order to estimate it and subtract it (intereference cancellation). The mutual information rate, call it $I$, between the interference signal and the channel output then gives some assessment concerning the quality of this estimation. For one thing, $D(I)$, where $D(\cdot)$ is the distortion-rate function of the source, is a lower bound to the distortion in estimating this signal. Moreover, if the channel is Gaussian, one can calculate the exact minimum mean square error (MMSE) from the mutual information rate $I$ by taking its derivative w.r.t. the signal-to-noise ratio (SNR) [9]. Another application comes from scenarios where the above described receiver is a hositle party (an eavesdropper), from which one would like to conceal information as much as possible. The natural setup, in this context, is that of the wiretap channel (cf. [14] as well as many follow-up papers), where excess channel noise beyond capacity is harnessed as an effective key that secures data communication. As we show in the sequel, the mutual information rate between the transmitted message and the eavesdropper, which suffers from this excess noise, is strongly related to the equivocation, which is a customary measure of security in Shannon-theoretic secrecy systems.

The outline of this paper is as follows. In Section 2, we establish notation conventions. In Section 3, we provide some basic background of elementary statistical physics, which will be needed in the sequel. In Section 4, we derive our main result, which is a formula for the mutual information rate. In Section 5, we demonstrate how it is applied for the wiretap channel, and finally, in Section 6 , we demonstrate how our results can be extended to multiuser scenarios, like that of the multiple access channel.

\section{Notation Conventions}

Throughout this paper, scalar random variables (RV's) will be denoted by the capital letters, like $S, X$, and $Y$, their sample values will be denoted by the respective lower case letters, and their alphabets will be denoted by the respective calligraphic letters. A similar convention will apply to 
random vectors and their sample values, which will be denoted with same symbols with the bold face font. Thus, for example, $\boldsymbol{X}$ will denote a random $n$-vector $\left(X_{1}, \ldots, X_{n}\right)$, and $\boldsymbol{x}=\left(x_{1}, \ldots, x_{n}\right)$ is a specific vector value in $\mathcal{X}^{n}$, the $n$-th Cartesian power of $\mathcal{X}$. Sources and channels will be denoted generically by the letter $P, Q, M$ and $W$. Whenever clarity and unambiguity will require it, these letters will be subscripted by the names of the relevant RV's, following the standard notation conventions in the literaure, for example, $P_{S}$ will denote the probability distribution of a random variable $S, P_{X \mid Y}$ will denote the conditional probability distribution of $X$ given $Y$, and so on. The cardinality of a finite set $\mathcal{A}$ will be denoted by $|\mathcal{A}|$. Information theoretic quantities like entropies and mutual informations will be denoted following the usual conventions of the information theory literature.

\section{Background}

In this section, we provide a brief account of the very basic background in statistical physics, which is needed for this paper.

Consider a physical system with $N$ of particles, which can be in a variety of microscopic states ('microstates'), defined by combinations of of physical quantities associated with these particles, e.g., positions, momenta, angular momenta, spins, etc., of all $N$ particles. For each such microstate of the system, which we shall designate by a vector $s=\left(s_{1}, \ldots, s_{N}\right)$, there is an associated energy, given by an Hamiltonian (energy function), $\mathcal{E}(\boldsymbol{s})$. For example, if $s_{i}=\left(\boldsymbol{p}_{i}, \boldsymbol{r}_{i}\right)$, where $\boldsymbol{p}_{i}$ is the momentum vector of particle number $i$ and $\boldsymbol{r}_{i}$ is its position vector, then classically,

$\mathcal{E}(\boldsymbol{s})=\sum_{i=1}^{N}\left[\frac{\left\|\boldsymbol{p}_{i}\right\|^{2}}{2 m}+m g z_{i}\right]$, where $m$ is the mass of each particle, $z_{i}$ is its height - one of the coordinates of $\boldsymbol{r}_{i}$, and $g$ is the gravitation constant.

One of the most fundamental results in statistical physics (based on the law of energy conservation and the basic postulate that all microstates of the same energy level are equiprobable) is that when the system is in thermal equilibrium with its environment, the probability of a microstate $s$ is given by the Boltzmann-Gibbs distribution

$$
P(s)=\frac{e^{-\beta \mathcal{E}(\boldsymbol{s})}}{Z(\beta)}
$$

where $\beta=1 /(k T), k$ being Boltmann's contant and $T$ being temperature, and $Z(\beta)$ is the normal- 
ization constant, called the partition function, which is given by

$$
Z(\beta)=\sum_{\boldsymbol{s}} e^{-\beta \mathcal{E}(\boldsymbol{s})}
$$

or

$$
Z(\beta)=\int d \boldsymbol{s} e^{-\beta \mathcal{E}(\boldsymbol{s})},
$$

depending on whether $s$ is discrete or continuous. The role of the partition function is by far deeper than just being a normalization factor, as it is actually the key quantity from which many macroscopic physical quantities can be derived, for example, the free energy ${ }^{1}$ is $-\frac{1}{\beta} \ln Z(\beta)$, the average internal energy (i.e., the expectation of $\mathcal{E}(s)$ where $s$ drawn is according (5)) is given by the negative derivative of $\ln Z(\beta)$, the heat capacity is obtained from the second derivative, etc. One of the ways to obtain eq. (5), is as the maximum entropy distribution under an energy constraint (owing to the second law of thermodynamics), where $\beta$ plays the role of a Lagrange multiplier that controls this energy level.

Let us define the quantity:

$$
\Omega_{N, \delta}(\epsilon)=|\{s:(\epsilon-\delta / 2) N \leq \mathcal{E}(s) \leq(\epsilon+\delta / 2) N\}|
$$

and let us assume that the limit

$$
\Sigma(\epsilon)=\lim _{\delta \rightarrow 0} \lim _{N \rightarrow \infty} \frac{\ln \Omega_{N, \delta}(\epsilon)}{N}
$$

exists and that $\Sigma(\epsilon)$ is a differentiable concave function. $\Sigma(\epsilon)$ is the entropy of the physical system in its statistical-mechanical definition. We will see shortly that it is intimately related to the Shannon entropy associated with the Boltzmann-Gibbs probablity distribution $P(s)$ defined above.

To see why the concavity assumption makes sense, note that at least when $P(s)$ is a product distribution (namely, when $\mathcal{E}(s)=\sum_{i} \mathcal{E}\left(s_{i}\right)$ ),

$$
\Omega_{N_{1}+N_{2}, \delta}\left(\frac{N_{1} \epsilon_{1}+N_{2} \epsilon_{2}}{N_{1}+N_{2}}\right) \geq \Omega_{N_{1}, \delta}\left(\epsilon_{1}\right) \cdot \Omega_{N_{2}, \delta}\left(\epsilon_{2}\right)
$$

since for every configuration $s$, where $N_{1} \leq N$ particles have total energy $N_{1} \epsilon_{1}$ and $N_{2}=N-$ $N_{1}$ particles have total energy $N_{2} \epsilon_{2}$, the total energy of all $N=N_{1}+N_{2}$ particles is obviously

\footnotetext{
${ }^{1}$ The free energy means the maximum work that the system can carry out in any process of fixed temperature. The maximum is obtained when the process is reversible (slow, quasi-static changes in the system).
} 
$N_{1} \epsilon_{1}+N_{2} \epsilon_{2}$, but the converse is not true since there are other ways to split the total energy of $N_{1} \epsilon_{1}+N_{2} \epsilon_{2}$ between the two complementary subsets of particles. Thus, taking the logarithm of both sides, dividing by $\left(N_{1}+N_{2}\right)$, then taking the limits of $N_{1}, N_{2} \rightarrow \infty$ such that $N_{1} / N_{2}$ tends to a given constant, and finally, taking the limit of $\delta \rightarrow 0$, one readily observes that $\Sigma(\epsilon)$ is concave. An argument of the same spirit can be exercised in somewhat more general situations, e.g., when $P(s)$ has a Markov structure (namely, the physical system has some nearest-neighbor interactions), though some more caution is required.

Denoting

$$
\psi(\beta)=\lim _{N \rightarrow \infty} \frac{1}{N} \ln \sum_{\boldsymbol{s}} \exp \{-\beta \mathcal{E}(\boldsymbol{s})\}
$$

it is readily seen that

$$
\begin{aligned}
\psi(\beta) & \left.=\lim _{\delta \rightarrow 0} \lim _{N \rightarrow \infty} \frac{1}{N} \ln \left[\sum_{j \geq 0} \Omega_{N, \delta}((j+1 / 2) \delta) \cdot \exp \{-N \beta j \delta]\right\}\right] \\
& =\sup _{\epsilon \geq 0}[\Sigma(\epsilon)-\beta \epsilon],
\end{aligned}
$$

i.e., $\psi(\cdot)$ and $\Sigma(\cdot)$ are a Legendre-transform pair. Since $\Sigma(\cdot)$ is assumed concave, then the inverse transform relation

$$
\Sigma(\epsilon)=\inf _{\beta \geq 0}[\beta \epsilon+\psi(\beta)]
$$

holds true as well, and so the derivatives $\beta(\epsilon) \triangleq d \Sigma / d \epsilon$ and $\epsilon(\beta)=-d \psi / d \beta$ (which are the maximizer of $[\Sigma(\epsilon)-\beta \epsilon]$ and the minimizer of $[\beta \epsilon+\psi(\beta)]$, respectively), are inverses of each other. It follows then that

$$
\Sigma(\epsilon)=\psi(\beta)-\beta \cdot \frac{d \psi}{d \beta},
$$

but as is readily seen, $-d \psi / d \beta$ is the average internal energy, $\boldsymbol{E}\{\mathcal{E}(\boldsymbol{S})]\}$, where $\boldsymbol{E}$ is the expectation operator associated with the Boltzmann distribution. This, in turn, is readily verified to agree with the expression of the Shannon entropy rate $H(S)$ of the distribution $P(s)$,

$$
\begin{aligned}
H(S) & =\lim _{n \rightarrow \infty} \frac{1}{N} \boldsymbol{E}\left\{\ln \left[\frac{1}{P(\boldsymbol{S})}\right]\right\} \\
& =\lim _{n \rightarrow \infty} \frac{1}{n} \boldsymbol{E}\left\{\ln \left[\frac{Z(\beta)}{\exp \{-\beta \mathcal{E}(\boldsymbol{S})\}}\right]\right\} \\
& =\psi(\beta)+\beta \boldsymbol{E}\{\mathcal{E}(\boldsymbol{S})\} .
\end{aligned}
$$

Thus, $\Sigma(\epsilon)=H(S)$ whenever $\beta$ and $\epsilon$ are related by $\beta=\beta(\epsilon)$, or equivalently, $\epsilon=\epsilon(\beta)$. For a given $\beta$, the Boltzmann-Gibbs distribution has a sharp peak (for large $N$ ) at the level of $\epsilon(\beta)$. We 
then say that this value of $\epsilon$ is the dominant energy level: Not only is it the average energy, there is also a strong concentration of the probability about this value as $N$ grows without bound. The second law of thermodynamics asserts that in an isolated system (which does not exchange energy with its environment), the total entropy cannot decrease, and hence in equilibrium, it reaches its maximum.

Now, suppose that we have a physical system that is composed of two subsystems, one having $N$ particles with microstates $\{\boldsymbol{s}\}$ and Hamiltonian $\mathcal{E}_{1}(\boldsymbol{s})$, and the other has $n$ particles with microstates $\left\{\boldsymbol{s}^{\prime}\right\}$ and Hamiltonian $\mathcal{E}_{2}\left(\boldsymbol{s}^{\prime}\right)$. Let us suppose that these two subsystems are in thermal contact and they both reside in a very large environment (heat bath) having a fixed temperature $T=1 /(k \beta)$. The two subsystems are allowed to exchange energy with each other as well as with the heat bath. How is the total energy of the system split between the two subsystems? An example of two such subsystems was described in the first few paragraphs of the Introduction.

The partition function of the composite system is given by

$$
Z(\beta)=\sum_{\boldsymbol{s}, \boldsymbol{s}^{\prime}} \exp \left\{-\beta\left[\mathcal{E}_{1}(\boldsymbol{s})+\mathcal{E}_{2}\left(\boldsymbol{s}^{\prime}\right)\right]\right\}
$$

and so the dominant energy level, as we saw before, is the one that achieves the associated normalized $\log$-partition function $\psi(\beta)$, i.e., the solution $\epsilon_{0}$ to the equation $d \Sigma(\epsilon) / d \epsilon=\beta$, where $\Sigma(\epsilon)$ is the entropy of the combined system. Let us confine attention now to the set of combined microstates $\left\{\left(\boldsymbol{s}, \boldsymbol{s}^{\prime}\right)\right\}$ of the composite system which have energy $(N+n) \epsilon_{0}$. More precisely, assume that the ratio $n / N=\lambda$ is held fixed, so $(N+n) \epsilon_{0}=N(1+\lambda) \epsilon_{0}$, and let us define

$$
\Omega_{N, n, \delta}\left(\epsilon_{0}\right)=\left|\left\{\left(s, s^{\prime}\right): N(1+\lambda)\left(\epsilon_{0}-\delta / 2\right) \leq \mathcal{E}_{1}(s)+\mathcal{E}_{2}\left(\boldsymbol{s}^{\prime}\right) \leq N(1+\lambda)\left(\epsilon_{0}+\delta / 2\right)\right\}\right| .
$$

Clearly, every configuration $\left(s, s^{\prime}\right)$ with energy about $N(1+\lambda) \epsilon_{0}$ corresponds to some allocation of of the energy in one subsystem and the remaining energy in the other. Thus, defining $\Omega_{N, \delta}^{(1)}(\epsilon)$ and $\Omega_{n, \delta}^{(2)}(\epsilon)$ as the enumerators of microstates with energy about $\epsilon$ in each one of the two subsystems individually (as defined in eq. (6)), we have, for $\hat{\delta}=\delta(1+\lambda)$ :

$$
\Omega_{N, n, \hat{\delta}}\left(\epsilon_{0}\right)=\sum_{j \geq 0} \Omega_{N, \delta}^{(1)}((j+1 / 2) \delta) \Omega_{n, \delta}^{(2)}\left(\frac{(1+\lambda) \epsilon_{0}-(j+1 / 2) \delta}{\lambda}\right) .
$$

Defining $\Sigma(\epsilon)$ as $\lim _{\delta \rightarrow 0} \lim _{N \rightarrow \infty}\left[\ln \Omega_{N, \lambda N, \hat{\delta}}(\epsilon)\right] /[N(1+\lambda)]$, we find, after taking logarithms of both sides, dividing by $N(1+\lambda)$, letting $N \rightarrow \infty$, and then $\delta \rightarrow 0$, that $\Sigma\left(\epsilon_{0}\right)$ is given by the weighted 
supremal convolution ${ }^{2}$ :

$$
\Sigma\left(\epsilon_{0}\right)=\sup _{0 \leq \epsilon \leq(1+\lambda) \epsilon_{0}}\left[\frac{1}{1+\lambda} \cdot \Sigma_{1}(\epsilon)+\frac{\lambda}{1+\lambda} \cdot \Sigma_{2}\left(\frac{(1+\lambda) \epsilon_{0}-\epsilon}{\lambda}\right)\right] .
$$

Assuming that the maximum is achieved by $\epsilon^{*} \in\left(0,(1+\lambda) \epsilon_{0}\right)$, it is characterized by a vanishing derivative of the expression in the square brackets, i.e., the solution to the equation

$$
\Sigma_{1}^{\prime}(\epsilon)=\Sigma_{2}^{\prime}\left(\frac{(1+\lambda) \epsilon_{0}-\epsilon}{\lambda}\right)
$$

where $\epsilon$ is the unknown, and where $\Sigma_{i}^{\prime}$ is the derivative of $\Sigma_{i}, i=1,2$. This equation characterizes the thermal equilibrium between the two subsystems and the heat bath. Now, the left-hand side is exactly $\beta$. Thus, $\epsilon^{*}$, the per-particle energy share of the first subsystem is the solution to the equation $\Sigma_{1}^{\prime}(\epsilon)=\beta$ (or, equivalently, of eq. (9), as said), and the remaining energy per particle, $\left[(1+\lambda) \epsilon_{0}-\epsilon^{*}\right] / \lambda$ belongs to the other subsystem.

Comment. Returning to the example that opens the Introduction, a simple calculation shows that the dominant energies are

$$
H \cdot \boldsymbol{E}\left\{\sum_{i=1}^{N} S_{i}\right\}=N B \tanh \left(\frac{B}{k T}\right)
$$

in the first subsystem, and

$$
e_{0} \cdot \boldsymbol{E}\left\{\sum_{i=1}^{n} S_{i}^{\prime}\right\}=\frac{n e_{0} \exp \left\{-e_{0} / k T\right\}}{1+\exp \left\{-e_{0} / k T\right\}}
$$

in the second subsystem. Thus,

$$
\epsilon^{*}=B \tanh \left(\frac{B}{k T}\right)
$$

and

$$
\frac{(1+\lambda) \epsilon_{0}-\epsilon^{*}}{\lambda}=\frac{\exp \left\{-e_{0} / k T\right\}}{1+\exp \left\{-e_{0} / k T\right\}} .
$$

In the parallel joint source-channel coding problem described in the Introduction, and to be further studied in a more general setting in the sequel, we have: $\ln P(s)=\left(\frac{1}{2} \ln \frac{q}{1-q}\right) \cdot \sum_{i=1}^{N} s_{i}+$ const, and $\ln W(\boldsymbol{y} \mid \boldsymbol{x})=\left(\ln \frac{p}{1-p}\right) \cdot \sum_{i=1}^{n}\left(x_{i} \oplus y_{i}\right)+$ const, with $\oplus$ denoting modulo 2 addition, the dominant contribution to $P(\boldsymbol{s} \mid \boldsymbol{y})$ comes from those $\{\boldsymbol{s}\}$ for which $\sum_{i=1}^{N} s_{i}$ is about its typical value $N[(+1) \cdot q+(-1) \cdot(1-q)]=N(2 q-1)=N \tanh (B / k T)$ (in analogy to the energy of the

\footnotetext{
${ }^{2}$ The supremal convolution between two functions $f(x)$ and $g(x)$ is generally defined as $h(x)=\sup _{t}[f(x-t)+g(t)]$. The qualifier "weighted", in our context, refers to the fact that both functions as well as their arguments are weighted by $1 /(1+\lambda)$ and $\lambda /(1+\lambda)$.
} 
first subsystem above, where we have used the relations (1)-(4)) and $\sum_{i=1}^{n}\left(x_{i} \oplus y_{i}\right)$ is about $n p=n \exp \left\{-e_{0} / k T\right\} /\left[1+\exp \left\{-e_{0} / k T\right\}\right]$ (in analogy to the energy of the second subsystem). Notice that these two typical contributions to the log-posterior probability agree also with the corresponding typical contributions, $\ln P\left(\boldsymbol{s}_{0}\right)$ and $\ln W\left(\boldsymbol{y} \mid \boldsymbol{x}\left(\boldsymbol{s}_{0}\right)\right)$, of the real message $\boldsymbol{s}_{0}$ that was actually transmitted. This is true regardless of whether the communication is reliable or not, i.e., it continues to hold no matter whether the entropy rate of the source is smaller or larger than $\lambda$ times the mutual information between the input and the output of the channel.

Returning to the general discussion above, note that the same considerations continue to hold even if one of the systems, say, the second one, has an effective negative entropy, that is, $\Omega_{n, \delta}^{(2)}([(1+$ $\left.\left.\lambda) \epsilon_{0}-\epsilon^{*}\right] / \lambda\right)<1$, which means that for each microstate $s$ of the first subsystem with per-particle energy $\epsilon^{*}$, only a fraction of the compatible combined microstates $\left\{\left(s, s^{\prime}\right)\right\}$ have noramilzed energy $\epsilon_{0}$. Of course, $\Omega_{N, n, \hat{\delta}}\left(\epsilon^{*}\right)$ must be larger than 1. In the sequel, we shall see that in the joint sourcechannel coding problem, the source and the channel constitute a mechanism which is highly parallel to that of equilibrium energy-sharing between two subsystems in a heat bath, where the subsystem corresponding to the channel has a negative effective thermodynamic entropy in this sense.

We should comment that in order to determine the energy sharing between the two subsystems in the above discussion, it was not necessary to consider how they thermally interact with each other and to go through the weighted supremal convolution between their entropies, as we did. We could have determined these energies simply by considering the equilibrium of each one of the subsystems individually with the heat bath, ${ }^{3}$ thus equating the derivative of each one of the entropy functions to $\beta$. Nonetheless, we have deliberately chosen to present the supremal convolution because in the sequel, it is this relation that will lead to the derivation of the mutual information in the joint source-channel coding problem.

\section{Formulation, Main Results and Discussion}

Consider an information source, $S_{1}, S_{2}, \ldots$, whose symbols $\left\{S_{i}\right\}$ take on values in a finite alphabet $\mathcal{S}$. The source is characterized by a sequence of probability distributions, $P(s), s \triangleq\left(s_{1}, \ldots, s_{N}\right)$, where $N=1,2, \ldots$. Consider next a discrete memoryless channel (DMC), which is characterized

\footnotetext{
${ }^{3}$ When doing so, the other system then becomes part of the heat bath anyway.
} 
by a matrix of single-letter transition probabilities $\{W(y \mid x), x \in \mathcal{X}, y \in \mathcal{Y}\}$, where $\mathcal{X}$ and $\mathcal{Y}$ are finite alphabets. The operation rate of the channel relative to the source is $\lambda$ channel uses per source symbol, which means that while the source produces an $N$-vector $s=\left(s_{1}, \ldots, s_{N}\right) \in \mathcal{S}^{N}$, the channel conveys $n$ channel symbols, namely, it receives an $n$-vector $\boldsymbol{x}=\left(x_{1}, \ldots, x_{n}\right) \in \mathcal{X}^{n}$ and outputs an $n$-vector $\boldsymbol{y}=\left(y_{1}, \ldots, y_{n}\right) \in \mathcal{Y}^{n}$, where $n=\lambda N$. The parameter $\lambda$ is referred to as the bandwidth expansion factor of the channel relative to the source.

For the sake of convenience in drawing the analogy with statistical mechanics, we will think of both the source and the channel as Boltzmann distributions with certain Hamiltonians at a certain common inverse temperature $\beta$, that is, $P(\boldsymbol{s})$ is proportional to $\exp \left\{-\beta \mathcal{E}_{S}(s)\right\}$ and $W(y \mid x)$ is proportional to $\exp \left\{-\beta \mathcal{E}_{C}(x, y)\right\}$, where $\mathcal{E}_{S}(\cdot)$ and $\mathcal{E}_{C}(\cdot, \cdot)$ are the Hamiltonians of the source and the channel, respectively. For a pair of $n$-vectors $\boldsymbol{x}$ and $\boldsymbol{y}$, we will denote $W(\boldsymbol{y} \mid \boldsymbol{x})=\prod_{i=1}^{n} W\left(y_{i} \mid x_{i}\right)$, and keep in mind that it is proportional to $\exp \left\{-\beta \mathcal{E}_{C}(\boldsymbol{x}, \boldsymbol{y})\right\}$, where $\mathcal{E}_{C}(\boldsymbol{x}, \boldsymbol{y}) \triangleq \sum_{i=1}^{n} \mathcal{E}_{C}\left(x_{i}, y_{i}\right)$. Clearly, there is no loss of generality in this representation of the source and the channel since there is always at least one way of doing this: For example, one can simply take $\beta=1, \mathcal{E}_{S}(s)=-\ln P(s)$, and $\mathcal{E}_{C}(x, y)=-\ln W(y \mid x)$. The point is, however, that by doing this we have slightly extended the scope: instead of one source and one channel, we are actually considering a family of sources and channels, both indexed by a common parameter $\beta$, that controls the degree of uniformity or skewedness of the distribution.

An $(N, n)$ joint source-channel code, for the above defined source and channel, is a mapping from the set $\mathcal{S}^{N}$ to $\mathcal{X}^{n}$. Every source string $s$ is mapped into a channel input vector $\boldsymbol{x} \triangleq\left(x_{1}, \ldots, x_{n}\right)$, and when we wish to emphasize the dependence of $\boldsymbol{x}$ on $\boldsymbol{s}$, we denote it as $\boldsymbol{x}(\boldsymbol{s})$. The code is assumed to be selected at random, where for each $\boldsymbol{s}$, the codeword $\boldsymbol{x}(\boldsymbol{s})$ is drawn under a distribution ${ }^{4} M(\boldsymbol{x})$, independently ${ }^{5}$ of all other codewords. The receiver estimates $s$ by applying a certain function on the received channel output sequence $\boldsymbol{y} \triangleq\left(y_{1}, \ldots, y_{n}\right)$, i.e., it implements a function from $\mathcal{Y}^{n}$ to $\mathcal{S}^{N}$, which will be denoted by $\hat{\boldsymbol{s}}=\hat{\boldsymbol{s}}(\boldsymbol{y})$. In some applications, the receiver (or the observer) may not necessarily attempt at full-fledged decoding of the message, but may opt to merely estimate a

\footnotetext{
${ }^{4}$ A more general model would allow a distribution $M$ that depends on $s$. For example, if $\mathcal{S}^{N}$ can be naturally divided into type classes (like in te case of memoryless sources, Markov sources, etc.), then it is plausible to let $M$ depend on the type class of $s$. However, among all sequences in $\mathcal{S}^{N}$, the important ones are those that are typical to the source (others can be ignored in the large $N$ limit), which are equiprobable in the exponential scale, and so, the distribution $M$ for all of them can be taken to be the same without loss of asymptotic optimality.

${ }^{5}$ The independence assumption is made here mostly for the sake of simplicity. It can be somewhat relaxed as long as the concentration properties specified below continue to hold.
} 
certain function of the source sequence (e.g., some statistic such as its composition).

Our study of the mutual information induced by the joint source-channel code will be strongly based on the posterior distribution, which, for a given (randomly selected) code, is defined as:

$$
\begin{aligned}
& P_{\beta}(\boldsymbol{s} \mid \boldsymbol{y})=\frac{P(\boldsymbol{s}) W(\boldsymbol{y} \mid \boldsymbol{x}(\boldsymbol{s}))}{\sum_{\boldsymbol{s}^{\prime} \in \mathcal{S}^{N}} P\left(\boldsymbol{s}^{\prime}\right) W\left(\boldsymbol{y} \mid \boldsymbol{x}\left(\boldsymbol{s}^{\prime}\right)\right)} \\
& =\frac{\exp \left\{-\beta\left[\mathcal{E}_{S}(\boldsymbol{s})+\mathcal{E}_{C}(\boldsymbol{x}(\boldsymbol{s}), \boldsymbol{y})\right]\right\}}{\sum_{\boldsymbol{s}^{\prime}} \exp \left\{-\beta\left[\mathcal{E}_{S}\left(\boldsymbol{s}^{\prime}\right)+\mathcal{E}_{C}\left(\boldsymbol{x}\left(\boldsymbol{s}^{\prime}\right), \boldsymbol{y}\right)\right]\right\}} .
\end{aligned}
$$

On a technical note, observe that since the posterior distribution is given by a ratio, this allows slighlty more freedom in the definition of the Hamiltonians $\mathcal{E}_{S}$ and $\mathcal{E}_{C}$, as certain common constants in the numerator and the denominator may cancel each other. For example, if the source is binary and memoryless, as described in the example given in the Introduction, then $P(s)$ is proportional to $\exp \left\{-\left(\frac{1}{2} \ln \frac{1-q}{q}\right) \sum_{i=1}^{N} s_{i}\right\}$, and so one can define $\mathcal{E}_{S}(s)$ to be proportional to $\sum_{i=1}^{N} s_{i}$, where the factor $\frac{1}{2} \ln \frac{1-q}{q}$ can be split between a part that is absrobed in the Hamiltonian itself and a part that is attributed to the inverse temperature parameter $\beta$. A similar comment applies to the channel, but here some more caution is required since, in general, the constant of proportionality that relates $W(\boldsymbol{y} \mid \boldsymbol{x})$ and $\exp \left\{-\beta \mathcal{E}_{C}(\boldsymbol{x}, \boldsymbol{y})\right\}$ may depend on $\boldsymbol{x}$, unless the code is of constant composition and/or the channel is symmetric in the sense that $\sum_{y} \exp \left\{-\beta \mathcal{E}_{C}(x, y)\right\}$ is independent of $x$ for all $\beta$ (which is the case, e.g., in modulo-additive channels, like the BSC). If neither of these conditions hold (i.e., if the code is not constant composition and the channel is not symmetric), we keep the choice $\mathcal{E}_{C}(x, y)$ as being proportional to $-\ln W(y \mid x)$.

For a given choice of the Hamiltonians $\mathcal{E}_{S}$ and $\mathcal{E}_{C}$, in view of these considerations, let us define the joint source-channel partition function as the denominator of the posterior distribution, i.e.,

$$
Z(\beta \mid \boldsymbol{y}) \triangleq \sum_{\boldsymbol{s} \in \mathcal{S}^{N}} \exp \left\{-\beta\left[\mathcal{E}_{S}(\boldsymbol{s})+\mathcal{E}_{C}(\boldsymbol{x}(\boldsymbol{s}), \boldsymbol{y})\right]\right\}
$$

In the course of studying the properties of a typical realization of the joint source-channel partition function, pertaining to a given code ensemble, we will make a few observations, which were already mentioned briefly in the Introduction:

1. Similarly as results that have already been observed in the context of the pure channel coding problem [12], the statistical-mechanical system pertaining to $Z(\beta \mid \boldsymbol{y})$ undergoes a phase transition, which corresponds, in the realm of coded systems, to the transition between reliable 
and unreliable communication, namely, the point at which the entropy rate of the source exceeds the mutual information between the input and the output of the channel.

2. When identifying the set of source vectors $\{s\}$ that dominates $Z(\beta \mid \boldsymbol{y})$ (i.e., those that contribute most to $Z(\beta \mid \boldsymbol{y}))$ above the phase transition temperature, one observes a situation that parallels that of thermal equilibrium between two physical subsystems, one corresponding to the source and the other corresponds to the channel. To be more specific, if $\mathcal{E}(\boldsymbol{s}, \boldsymbol{y})=\mathcal{E}_{S}(\boldsymbol{s})+\mathcal{E}_{C}(\boldsymbol{x}(\boldsymbol{s}), \boldsymbol{y})$ is thought of as the total 'energy' shared by the source and the code/channel, then the dominant messages $\{s\}$ split this total average energy between the source and the channel components in a way that corresponds to thermal equilibrium between the two parallel physical subsystems.

3. The balance between the thermodynamical entropies of the two physical subsystems that lie in equilibrium, as described in item no. 2 , is identified with the simple relation between the corresponding Shannon entropies of the source, namely, the unconditional source entropy and the conditional entropy given the channel output, whose difference is the mutual information between the source and the channel output. This gives rise to a simple formula of the mutual information rate induced by a typical code in the ensemble.

In analogy to the definitions and the assumptions outlined in Section 3, we now make a few definitions and assumptions concerning the joint source-channel coding model.

A.1 Defining

$$
\Omega_{N, \delta}^{(S)}(\epsilon) \triangleq\left|\left\{s \in \mathcal{S}^{N}:(\epsilon-\delta / 2) N \leq \mathcal{E}_{S}(s) \leq(\epsilon+\delta / 2) N\right\}\right|
$$

our first assumption is that

$$
\Sigma_{S}(\epsilon) \triangleq \lim _{\delta \rightarrow 0} \lim _{N \rightarrow \infty} \frac{\ln \Omega_{N, \delta}^{(S)}(\epsilon)}{N}
$$

exists and that $\Sigma_{S}(\epsilon)$ is a differentiable concave function.

A.2 For a given $\boldsymbol{y}$, define

$$
\phi_{n, \delta}(\epsilon \mid \boldsymbol{y}) \triangleq \frac{1}{n} \ln \operatorname{Pr}\left\{n(\epsilon-\delta / 2) \leq \mathcal{E}_{C}(\boldsymbol{X}, \boldsymbol{y}) \leq n(\epsilon+\delta / 2)\right\},
$$

where the random vector $\boldsymbol{X}$ is drawn under the random coding distribution $M$, independently of $\boldsymbol{y}$. Then, our second assumption is that for all $\epsilon \geq 0, \lim _{\delta \rightarrow 0} \lim _{n \rightarrow \infty} \boldsymbol{E}\left\{\phi_{n, \delta}(\epsilon \mid \boldsymbol{Y})\right\}$ tends 
uniformly to a differentiable function $\phi(\epsilon)$, where the expectation $\boldsymbol{E}$ is w.r.t. both the random selection of the codebook and the random actions of the source and the channel. Moreover, we assume that $\lim _{\delta \rightarrow 0} \lim _{n \rightarrow \infty} \phi_{n, \delta}(\epsilon \mid \boldsymbol{Y})$ tends $\phi(\epsilon)$ uniformly almost surely.

A.3 Let $\Sigma_{S}(\epsilon)$ and $\phi(\epsilon)$ be defined as above, and let $\Sigma_{0}(\epsilon)$ be defined by the weighted supremal convolution

$$
\Sigma_{0}(\epsilon) \triangleq \max _{0 \leq \epsilon^{\prime} \leq(1+\lambda) \epsilon}\left[\frac{\Sigma_{S}\left(\epsilon^{\prime}\right)}{1+\lambda}+\frac{\lambda}{1+\lambda} \phi\left(\frac{(1+\lambda) \epsilon-\epsilon^{\prime}}{\lambda}\right)\right] .
$$

Our third assumption is that $\Sigma_{0}(\epsilon)$ is a concave function throughout the range of $\epsilon$ where it is non-negative. We now define

$$
\Sigma(\epsilon)= \begin{cases}\Sigma_{0}(\epsilon) & \Sigma_{0}(\epsilon) \geq 0 \\ -\infty & \Sigma_{0}(\epsilon)<0\end{cases}
$$

As we shall see below, while $\Sigma_{0}(\epsilon)$ gives the logarithm of the expected number of configurations with total energy $\epsilon$, the function $\Sigma(\epsilon)$ gives the number of such configurations for a typical code in the ensemble. To see this, note that if $\Sigma_{S}\left(\epsilon^{\prime}\right)+\lambda \phi\left(\left[(1+\lambda) \epsilon-\epsilon^{\prime}\right] / \lambda\right)<0$ for all $\epsilon^{\prime}$, this means that for every $\epsilon^{\prime}$ the product of the number of configurations $\{s\}$ for which $\mathcal{E}_{S}(s)$ is about $n \epsilon^{\prime}$ and the probability that a randomly chosen codeword would provide the complementary energy $\left(\left[(1+\lambda) \epsilon-\epsilon^{\prime}\right] / \lambda\right.$, is less than one, which means that there is a very low probability to find any configuration with total energy $\epsilon$, and so, $\Sigma(\epsilon)$ which is the normalized logarithm of the number of such configurations (i.e., the thermodynamical entropy of the combined system) is equal to $-\infty$ for a typical code realization. Note that the concavity of $\Sigma_{0}(\epsilon)$ across the range where it is non-negative implies that $\Sigma(\epsilon)$ is concave as well.

In analogy to the discussion of the previous section, let us define

$$
Z_{S}(\beta) \triangleq \sum_{\boldsymbol{s}} \exp \left\{-\beta \mathcal{E}_{S}(\boldsymbol{s})\right\}
$$

Then,

$$
\psi_{S}(\beta) \triangleq \lim _{N \rightarrow \infty} \frac{1}{N} \ln Z_{S}(\beta)
$$

and $\Sigma_{S}(\epsilon)$ are a Legendre-transform pair. Since $\Sigma_{S}(\cdot)$ is assumed concave, then the inverse transform relation

$$
\Sigma_{S}(\epsilon)=\inf _{\beta \geq 0}\left[\beta \epsilon+\psi_{S}(\beta)\right]
$$


holds true as well, and so the derivatives $\beta_{S}(\epsilon) \triangleq d \Sigma_{S} / d \epsilon$ and $\epsilon_{S}(\beta)=-d \psi_{S} / d \beta$ are inverses of each other. It follows then that the Shannon entropy rate $H(S)$ of $P(s)$ (which depends on $\beta$ ) agrees with $\Sigma_{S}(\epsilon)$ whenever $\beta$ and $\epsilon$ are related by $\beta=\beta_{S}(\epsilon)$, or equivalently, $\epsilon=\epsilon_{S}(\beta)$.

Referring to the partition function $Z(\beta \mid \boldsymbol{y})$, let us distinguish between the contribution of the actual realization of the true sequence that the source actually emitted $\boldsymbol{s}_{0}$, i.e., $Z_{c}(\beta \mid \boldsymbol{y})=$ $\exp \left\{-\beta\left[\mathcal{E}_{S}\left(\boldsymbol{s}_{0}\right)+\mathcal{E}_{C}\left(\boldsymbol{x}\left(\boldsymbol{s}_{0}\right), \boldsymbol{y}\right)\right]\right\}$ and the contribution of all other (erroneous) source vectors

$$
Z_{e}(\beta \mid \boldsymbol{y})=\sum_{\boldsymbol{s} \neq \boldsymbol{s}_{0}} \exp \left\{-\beta\left[\mathcal{E}_{S}(\boldsymbol{s})+\mathcal{E}_{C}(\boldsymbol{x}(\boldsymbol{s}), \boldsymbol{y})\right]\right\}
$$

Now, $\ln Z_{c}(\beta \mid \boldsymbol{y})$ is typically around $-\left[\boldsymbol{E}\left\{\mathcal{E}_{S}(\boldsymbol{S})\right\}+\boldsymbol{E}\left\{\mathcal{E}_{C}(\boldsymbol{X}(\boldsymbol{S}), \boldsymbol{Y})\right\}\right]$. As for $Z_{e}(\beta \mid \boldsymbol{y})$, let us define

$$
\Omega_{N, \delta}(\epsilon \mid \boldsymbol{y})=\left|\left\{\boldsymbol{s} \neq \boldsymbol{s}_{0}: N(1+\lambda)(\epsilon-\delta / 2) \leq \mathcal{E}_{S}(\boldsymbol{s})+\mathcal{E}_{C}(\boldsymbol{x}(\boldsymbol{s}), \boldsymbol{y}) \leq N(1+\lambda)(\epsilon+\delta / 2)\right\}\right|
$$

Then, similarly as in the previous section, one readily observes that for $\delta^{\prime}=\delta(1+\lambda)$, we have:

$$
\begin{aligned}
\Omega_{N, \delta^{\prime}}(\epsilon \mid \boldsymbol{y})= & \sum_{j \geq 0} \Omega_{N, \delta}^{(S)}((j+1 / 2) \delta) \times \\
& \left.\operatorname{Pr}\left\{N(1+\lambda)\left(\epsilon-\delta^{\prime} / 2\right)-N(j+1) \delta \leq \mathcal{E}_{C}(\boldsymbol{X}, \boldsymbol{y})\right) \leq N(1+\lambda)\left(\epsilon+\delta^{\prime} / 2\right)-N j \delta\right\} \\
= & \sum_{j \geq 0} \Omega_{N, \delta}^{(S)}((j+1 / 2) \delta) \exp \left\{n \phi_{n, \delta}([(1+\lambda) \epsilon-(j+1 / 2) \delta] / \lambda \mid \boldsymbol{y})\right\}
\end{aligned}
$$

Taking logarithms of both sides, dividing by $N+n=N(1+\lambda)$, letting $N$ grow without bound, and finally letting $\delta$ go to zero, we obtain ${ }^{6}$ that:

$$
\lim _{N \rightarrow \infty} \frac{\ln \hat{\Omega}_{N, \delta^{\prime}}(\epsilon \mid \boldsymbol{Y})}{N(1+\lambda)} \stackrel{\text { a.s. }}{=} \begin{cases}\Sigma_{0}(\epsilon) & \Sigma_{0}(\epsilon) \geq 0 \\ -\infty & \Sigma_{0}(\epsilon)<0\end{cases}
$$

but the r.h.s. is exactly $\Sigma(\epsilon)$. Thus, as explained earlier, $\Sigma(\epsilon)$ is the thermodynamical entropy associated with the combined source-channel system. The concavity of $\Sigma(\epsilon)$ then implies that it agrees (after the appropriate scaling) with the conditional Shannon entropy rate of the source given the channel output, $H(S \mid Y)$, i.e., the entropy rate pertaining to the sequence of conditional probabilities $P(\boldsymbol{s} \mid \boldsymbol{y})$ defined above. For a given $\epsilon$ in the range where $\Sigma(\epsilon)$ is finite, let $\epsilon^{\prime}=\epsilon^{*}$ achieve the supremum defining $\Sigma(\epsilon)$.

\footnotetext{
${ }^{6}$ At this point, we are using the fact [12],[11] that for an ensemble of independently selected codewords, the number of codewords which contribute energy $\mathcal{E}_{C}(\boldsymbol{X}, \boldsymbol{y}) \approx n\left[(1+\lambda) \epsilon-\epsilon^{\prime}\right] \lambda$, is with very high probability zero, if $\left.\left.\Sigma_{S}\left(\epsilon^{\prime}\right)+\lambda \phi(1+\lambda) \epsilon-\epsilon^{\prime}\right] / \lambda\right)<0$ and around $\left.\exp \left\{N\left[\Sigma_{S}\left(\epsilon^{\prime}\right)+\lambda \phi(1+\lambda) \epsilon-\epsilon^{\prime}\right] / \lambda\right)\right\}$ if $\left.\left.\Sigma_{S}\left(\epsilon^{\prime}\right)+\lambda \phi(1+\lambda) \epsilon-\epsilon^{\prime}\right] / \lambda\right)>0$. The assumption of independnent codewords can be relaxed as long as this concentration property continues to hold.
} 
At this point, one should distinguish between two situations: In the first situation, $\epsilon$ is on the boundary of the range where $\Sigma(\epsilon)$ is finite and positive, namely, $\Sigma(\epsilon)=0$. In this case, the partition function $Z(\beta \mid \boldsymbol{y})$ (and hence also $P_{\beta}(\boldsymbol{s} \mid \boldsymbol{y})$ ) is dominated by a subexponential number of configurations $\{s\}$ and so, the entropy rate $H(S \mid Y)=0$, which means that the system is frozen in its glassy phase (cf. [12],[11] and references therein.) In the second situation, $\epsilon$ is an internal point of the range where $\Sigma(\epsilon)>0$, where we will also assume that $\epsilon^{*} \in(0,(1+\lambda) \epsilon)$, which is the paramagnetic phase (or the disordered phase) of $Z_{e}(\beta \mid \boldsymbol{y})$. Then, the derivative of the function being maximized vanishes, i.e.,

$$
\left.\frac{d \Sigma_{S}\left(\epsilon^{\prime}\right)}{d \epsilon^{\prime}}\right|_{\epsilon^{\prime}=\epsilon^{*}}-\left.\frac{d \phi\left(\epsilon^{\prime \prime}\right)}{d \epsilon^{\prime \prime}}\right|_{\epsilon^{\prime \prime}=\left[(1+\lambda) \epsilon-\epsilon^{*}\right] / \lambda}=0
$$

or equivalently,

$$
\Sigma_{S}^{\prime}\left(\epsilon^{*}\right)=\phi^{\prime}\left(\frac{(1+\lambda) \epsilon-\epsilon^{*}}{\lambda}\right)
$$

where $\Sigma_{S}^{\prime}$ and $\phi^{\prime}$ denote the derivatives of $\Sigma_{S}$ and $\phi$, respectively. As before, eq. (12) gives rise to thermal equilbrium between the physical system corresponding to the source and the one that pertains to the code/channel. Next observe that the left-hand side is exactly $\beta_{S}\left(\epsilon^{*}\right)$. Thus,

$$
\beta_{S}\left(\epsilon^{*}\right)=\phi^{\prime}\left(\frac{(1+\lambda) \epsilon-\epsilon^{*}}{\lambda}\right)
$$

which means that given the value of the total per-particle energy $\epsilon$, we can find how the dominant codewords split the energy between the source and the channel: we can solve the above equation with the given $\epsilon$, with $\epsilon^{*}$ as an unknown. Then, the source contribution will be $\epsilon^{*}$ and the channel contribution will be $\left[(1+\lambda) \epsilon-\epsilon^{*}\right] / \lambda$.

The discussion above holds for every value of $\epsilon$ for which $\Sigma(\epsilon)>0$. The dominant value of $\epsilon$ is $\epsilon_{0}$, the one that achieves $\boldsymbol{E}\{\ln Z(\beta \mid \boldsymbol{Y})\} /[N(1+\lambda)]$ for large $N$, in other words, the achiever of:

$$
\psi(\beta)=\lim _{N \rightarrow \infty} \frac{\boldsymbol{E} \ln Z(\beta \mid \boldsymbol{Y})}{N(1+\lambda)}=\sup _{\epsilon \geq 0}[\Sigma(\epsilon)-\beta \epsilon] .
$$

Thus, the dominant value of $\epsilon$, which is relevant for the previous paragraph, is $\epsilon_{0}$, which in turn depends only on $\beta$. But since $\Sigma$ is assumed concave, then $\psi$ and $\Sigma$ are also a Legendre-transform pair, and so $\epsilon_{0}$ and $\beta$ are related via the derivatives, $\epsilon_{0}=\epsilon(\beta) \triangleq-\psi^{\prime}(\beta)$ and $\beta=\beta(\epsilon)=\Sigma^{\prime}(\epsilon)$, where again, primes denote the derivatives. In summary, given $\beta, \epsilon_{0}=\epsilon(\beta)$ and $\epsilon^{*}=\epsilon_{S}(\beta)$. Thus, 
$\beta_{S}\left(\epsilon^{*}\right)$ in the equilibrium equation is $\beta_{s}\left(\epsilon_{S}(\beta)\right) \equiv \beta$ since $\beta_{S}(\cdot)$ and $\epsilon_{S}(\cdot)$ are inverses of one another. Thus, the equilibrium equation applied to the dominant energy $\epsilon_{0}$ becomes

$$
\beta=\Sigma_{S}^{\prime}\left(\epsilon^{*}\right)=\phi^{\prime}\left(\frac{(1+\lambda) \epsilon_{0}-\epsilon^{*}}{\lambda}\right) .
$$

If, in addition, $\phi$ is concave, then $\phi^{\prime}$ is monotone, and thus has an inverse, which is given by the negative derivative $-\zeta^{\prime}$ of the Legendre transform of $\phi$, that is, by the derivative of

$$
\zeta(t)=\sup _{\epsilon}[\phi(\epsilon)-\epsilon t]
$$

and then

$$
\frac{(1+\lambda) \epsilon_{0}-\epsilon^{*}}{\lambda}=-\zeta^{\prime}(\beta)
$$

Now observe that if, for a typical $\boldsymbol{y}$, either $Z_{c}(\beta \mid \boldsymbol{y})$ dominates $Z_{e}(\beta \mid \boldsymbol{y})$, or $Z_{e}(\beta \mid \boldsymbol{y})$ is in its frozen phase, then $H(S \mid Y)$ vanishes, and so the mutual information rate $\lim _{N \rightarrow \infty} I(\boldsymbol{S} ; \boldsymbol{Y}) / N=H(S)$. For the complementary case, our main result is the following:

Theorem 1 Let $\boldsymbol{E}\{I(\boldsymbol{S} ; \boldsymbol{Y})\}$ denote the expected mutual information, where the expectation is taken w.r.t. the ensemble of of joint source-channel codes. Then, under Assumptions A1-A3:

$$
\lim _{N \rightarrow \infty} \frac{\boldsymbol{E}\{I(\boldsymbol{S} ; \boldsymbol{Y})\}}{N}=-\lambda \phi\left(-\zeta^{\prime}(\beta)\right),
$$

provided that $\Sigma\left(\epsilon_{0}\right)>0$.

Remark: From the above discussion, it is apparent that this result applies also to the almost-sure limit of $I(\boldsymbol{S} ; \boldsymbol{Y}) / N$ w.r.t. the code ensemble.

Proof.

$$
\begin{aligned}
\lim _{N \rightarrow \infty} \frac{\boldsymbol{E} I(\boldsymbol{S} ; \boldsymbol{Y})}{N} & =H(S)-H(S \mid Y) \\
& =\Sigma_{S}\left(\epsilon^{*}\right)-(1+\lambda) \Sigma\left(\epsilon_{0}\right) \\
& =-\lambda \phi\left(\frac{(1+\lambda) \epsilon_{0}-\epsilon^{*}}{\lambda}\right) \\
& =-\lambda \phi\left(-\zeta^{\prime}(\beta)\right) .
\end{aligned}
$$

Discussion. We obtained then a very simple formula which depends solely on the random coding distribution. But what is the meaning of $\zeta^{\prime}(\beta)$ ? Since $-\phi(\epsilon)$ is, in fact, the large deviations rate 
function for the event $\mathcal{E}_{C}(\boldsymbol{X}, \boldsymbol{y}) \leq n \epsilon$, and $\zeta(t)$ is its Legendre transform, then it must be the almost-sure limit of the log-moment generating function, that is

$$
\zeta(t) \stackrel{\text { a.S. }}{=} \lim _{n \rightarrow \infty} \frac{1}{n} \ln \sum_{\boldsymbol{x} \in \mathcal{X}^{n}} M(\boldsymbol{x}) e^{-t \mathcal{E}_{C}(\boldsymbol{x}, \boldsymbol{Y})}
$$

where, as defined above, $M$ is the random coding distribution that governs each one of the independent, randomly selected codewords. Thus,

$$
-\zeta^{\prime}(\beta) \stackrel{\text { a.s. }}{=} \lim _{n \rightarrow \infty} \frac{1}{n} \cdot \frac{\left.\sum \boldsymbol{x} M(\boldsymbol{x}) \mathcal{E}_{c}(\boldsymbol{x}, \boldsymbol{Y}) e^{-\beta \mathcal{E}_{C}(\boldsymbol{x}, \boldsymbol{Y})}\right\}}{\sum \boldsymbol{x} M(\boldsymbol{x}) e^{-\beta \mathcal{E}_{C}(\boldsymbol{x}, \boldsymbol{Y})}} .
$$

But the Boltzmann weight $e^{-\beta \mathcal{E}_{C}(\boldsymbol{x}, \boldsymbol{y})}$ is proportional to $W(\boldsymbol{y} \mid \boldsymbol{x})$, and so, $-\zeta^{\prime}(\beta)$ is exactly the asymptotic almost-sure normalized conditional expectation of the energy, $\lim _{n \rightarrow \infty} \boldsymbol{E}\left\{\mathcal{E}_{C}(\boldsymbol{X}, \boldsymbol{Y}) \mid \boldsymbol{Y}\right\} / n$, stemming from the action of the channel on the message $\boldsymbol{x}\left(\boldsymbol{s}_{0}\right)$ that was actually transmitted. This quantity in turn is assumed to concentrate about its mean which is $\lim _{n \rightarrow \infty} \boldsymbol{E}\left\{\mathcal{E}_{C}(\boldsymbol{X}, \boldsymbol{Y})\right\} / n$. Thus, $Z_{e}(\beta \mid \boldsymbol{y})$ and $P(\boldsymbol{s} \mid \boldsymbol{y})$ are dominated by (erroneous) sequences $\{\boldsymbol{s}\}$ whose normalized energy $\epsilon_{0}$ consists of a source contribution $\epsilon^{*}=\lim _{N \rightarrow \infty} \boldsymbol{E}\left\{\mathcal{E}_{S}(\boldsymbol{S})\right\} / N$, and a channel contribution, $\left[(1+\lambda) \epsilon_{0}-\epsilon^{*}\right] / \lambda$ that agrees with the normalized energy generated by the noise, i.e., it agrees with $\lim _{n \rightarrow \infty} \boldsymbol{E}\left\{\mathcal{E}_{C}(\boldsymbol{X}, \boldsymbol{Y})\right\} / n$, where $\boldsymbol{X}$ and $\boldsymbol{Y}$ are related via the channel $W$. Moreover, this is also the typical energy composition of the true message $s_{0}$ that was actually transmitted (cf. the definition of $Z_{c}(\beta \mid \boldsymbol{y})$. Thus, the above conclusion holds true regardless of whether or not the entropy rate of the source is smaller (in which case $\boldsymbol{s}_{0}$ dominates $Z(\beta \mid \boldsymbol{y})$ ) or larger than $\lambda$ times the normalized mutual informtion between $\boldsymbol{X}$ and $\boldsymbol{Y}$ (in which case, erroneous messages dominate $Z(\beta \mid \boldsymbol{y})$ for a typical $\boldsymbol{y}$ ). We have already seen this behavior in the special case of the binary source and the BSC.

Example 1. Suppose that the channel is BSC and codewords are generated by fair coin tossing. In this case, $W(\boldsymbol{y} \mid \boldsymbol{x})$ is proportional to $\exp \left\{-\beta \mathcal{E}_{C}(\boldsymbol{x}, \boldsymbol{y})\right\}$, where $\mathcal{E}_{C}(\boldsymbol{x}, \boldsymbol{y})$ is the Hamming distance and $\beta=\ln \frac{1-p}{p}$. In this case, $\phi(\epsilon)=h_{2}(\epsilon)-\ln 2$ whose derivative is $\phi^{\prime}(\epsilon)=\ln \frac{1-p}{p}$, and so, $-\zeta^{\prime}(\beta)$, the inverse of $\phi^{\prime}(\epsilon)$, is given by $-\zeta^{\prime}(\beta)=1 /\left(1+e^{\beta}\right)=p$. It follows then that if, in addition, the source is binary and memoryless with a parameter $q$, then $P(\boldsymbol{s} \mid \boldsymbol{y})$ is dominated by vectors $\{s\}$ whose energy is as described in the Introduction. Also, the normalized mutual information is $-\lambda \phi\left(-\zeta^{\prime}(\beta)\right)=-\lambda \phi(p)=\lambda\left(\ln 2-h_{2}(p)\right)$. Somewhat more generally, let each coordinate $X_{i}(s)$, $i=1, \ldots, n$, of each codeword be drawn i.i.d. with probabilities $\operatorname{Pr}\left\{X_{i}(\boldsymbol{s})=1\right\}=1-\operatorname{Pr}\left\{X_{i}(\boldsymbol{s})=\right.$ 
$0\}=m$. Then, it is easy to show (using the method of types [2]) that

$$
-\phi(p)=\min _{\left\{P_{X \mid Y}: \boldsymbol{E}_{d(X, Y) \leq p\}}\right.}\left[I(X ; Y)+D\left(P_{X} \| M\right)\right], \quad Y \sim \operatorname{Bernoulli}(m * p),
$$

where $m * p$ means the binary convolution between $m$ and $p$ (i.e., $m * p=m(1-p)+p(1-m)), d(\cdot, \cdot)$ is the Hamming distance and $P_{X}$ is the marginal of $X$ induced by $Y$ (which is Bernoulli $(m * p)$ ) and the reversed channel $P_{X \mid Y}$ to be optimized. By eliminating the divergence term, we are lower bounding $-\phi(p)$ by the rate-distortion function of $Y$ at Hamming distortion $p$, which is $h_{2}(m * p)-h_{2}(p)$. On the other hand, returning to the original minimization problem, by selecting $P_{X \mid Y}$ (instead of minimizing over $P_{X \mid Y}$ ) to be the reverse channel induced by $M$ and $W_{Y \mid X}$ (which is the $\operatorname{BSC}(p)$ ), we are getting the same quantity also as an upper bound. Thus, $-\phi(p)=h_{2}(m * p)-h_{2}(p)$, and so,

$$
\lim _{N \rightarrow \infty} \frac{\boldsymbol{E} I(\boldsymbol{S} ; \boldsymbol{Y})}{N}=\lambda\left[h_{2}(m * p)-h_{2}(p)\right]
$$

Comment: An alternative view on the derivation of the asymptotic mutual information rate between $\boldsymbol{S}$ and $\boldsymbol{Y}$ comes from the following chain of equalities:

$$
\begin{aligned}
\lim _{N \rightarrow \infty} \frac{\boldsymbol{E} I(\boldsymbol{S} ; \boldsymbol{Y})}{N}= & \lim _{N \rightarrow \infty} \boldsymbol{E}\left\{\ln \frac{P(\boldsymbol{Y} \mid \boldsymbol{S})}{P(\boldsymbol{Y})}\right\} \\
= & \lim _{N \rightarrow \infty} \frac{1}{N} \boldsymbol{E}\left\{\ln \exp \left\{-\beta \mathcal{E}_{C}(\boldsymbol{X}(\boldsymbol{S}), \boldsymbol{Y})\right\}\right\}- \\
& \lim _{N \rightarrow \infty} \frac{1}{N} \boldsymbol{E}\left\{\ln \left[\sum_{\boldsymbol{s}} \frac{1}{Z_{S}(\beta)} \exp \left\{-\beta\left[\mathcal{E}_{S}(\boldsymbol{s})+\mathcal{E}_{C}(\boldsymbol{X}(\boldsymbol{s}), \boldsymbol{Y})\right]\right\}\right]\right\} \\
= & -\beta\left[(1+\lambda) \epsilon_{0}-\epsilon^{*}\right]+\psi_{S}(\beta)-\Sigma_{S}\left(\epsilon^{*}\right)-\lambda \phi\left(\frac{\left.(1+\lambda) \epsilon_{0}-\epsilon^{*}\right)}{\lambda}\right)+\beta(1+\lambda) \epsilon_{0} \\
= & \beta \epsilon^{*}+\psi_{S}(\beta)-\Sigma_{S}\left(\epsilon^{*}\right)-\lambda \phi\left(\frac{\left.(1+\lambda) \epsilon_{0}-\epsilon^{*}\right)}{\lambda}\right) \\
= & -\lambda \phi\left(\frac{\left.(1+\lambda) \epsilon_{0}-\epsilon^{*}\right)}{\lambda}\right)
\end{aligned}
$$

where we have used the fact that the summation over $s$ is dominated by configurations with perparticle energy $\epsilon_{0}$, which is allocated as $\epsilon^{*}$ and $\left[(1+\lambda) \epsilon_{0}-\epsilon^{*}\right] / \lambda$.

\section{Application to the Wiretap Channel}

In this section, we demonstrate how our results apply to the wiretap channel. Wyner, in his wellknown paper on the wiretap channel [14], studied the problem of secure communication across a 
degraded broadcast channel, without using a secret key, where the legitimate receiver has access to the output of the good channel and the wiretapper receives the output of the bad channel. In that paper, Wyner characterized the optimum trade-off between reliable coding rates and the equivocation at the wiretapper, which was defined in terms of the conditional entropy of the source given the output of the bad channel, observed by the wire-tapper.

Consider a DMS $P$ as before, and a cascade of two finite alphabet DMC's: $W_{Y \mid X}$ followed immediately by $W_{Z \mid Y}$, both ${ }^{7}$ operating at a relative rate of $\lambda$ channel symbols per source symbol. The source $s \in \mathcal{S}^{N}$ is encoded to a channel input vector $\boldsymbol{x}(\boldsymbol{s}) \in \mathcal{X}^{n}, n=\lambda N$, and then transmitted. A code for the wire-tap channel should be designed in a way, that on the one hand, the legitimate receiver is required to estimate the source $s$ from the output $\boldsymbol{y} \in \mathcal{Y}^{n}$ of the channel $W_{Y \mid X}$ within an arbitrarily small probability of error, whereas on the other hand, the eavesdropper, which has access to $z \in \mathcal{Z}^{n}$, should be able to learn as little as possible about the source in the sense that

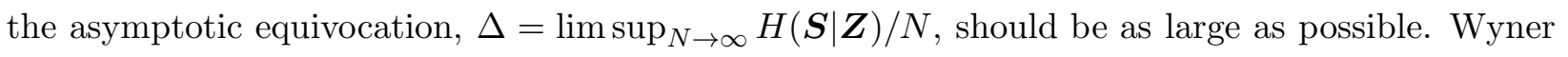
showed [14] that the largest achievable value of $\Delta$ is given by $\lambda \Gamma(H(S) / \lambda)$, where

$$
\Gamma(R) \triangleq \max _{P_{X}: I(X ; Y) \geq R}[I(X ; Y)-I(X ; Z)]
$$

In particular, the secrecy capacity $C_{s}$, which is the solution to the equation $R=\Gamma(R)$, is the rate at which the potential secrecy that the wiretap channel can offer is fully expoilted: If the entropy of the source, $H(S) / \lambda$ is less than or equal to $C_{s}$ (supposing that $\lambda$ can be chosen in such a way), then the coding scheme of [14] that asymptotically achieves $C_{s}$ works as follows: Let $X^{*}$ be the random variable $X$ that achieves $\Gamma(R)$, for some $R$ in the range $H(S) / \lambda \leq R \leq C_{s}$, and let $Y^{*}$ and $Z^{*}$ be the corresponding outputs of the two channels. We first compress the source $\boldsymbol{S}$ to its entropy, and then apply channel coding so that the good receiver can still decode reliably for large $N$ and $n$, but the bad one cannot. Now, since $H(S) / \lambda \leq C_{s}$, then by the definitions of $\Gamma(\cdot)$ and $C_{s}, I\left(X^{*} ; Y^{*}\right) \geq H(S) / \lambda+I\left(X^{*} ; Z^{*}\right)$. Accordingly, the channel codebook is composed of about $e^{N H(S)}=e^{n H(S) / \lambda}$ bins (one for each typical source sequence), each of size slightly less than $e^{n I\left(X^{*} ; Z^{*}\right)}$. The codeword actually transmitted is randomly chosen among all codewords of the bin pertaining to the index of the compressed source sequence. Note that the eavesdropper could have decoded the message had it been informed of the bin which the transmitted codeword

\footnotetext{
${ }^{7}$ The notation of the output of the second channel, $Z$, should not be confused with the notation of the partition function since we do not refer the partition function in this section.
} 
belongs to since the rate of the bin, as said, is (slightly) less than $I\left(X^{*} ; Z^{*}\right)$. The idea then is that this information is irrelevant since it is independent of the source vector, and so it does not help the eavesdropper in learning anything about the source. Indeed, if we represent the transmitted codeword $\boldsymbol{x}$ as $f(c(\boldsymbol{s}), \boldsymbol{u})$, where $c(\boldsymbol{s})$ stands for the bit string of the lossless compression of $\boldsymbol{s}$, indicating the bin index using $n H(S) / \lambda$ nats, and $\boldsymbol{u}$ as an independent random bit string of length $n I\left(X^{*} ; Z^{*}\right)$ nats, then we have the following: One the one hand,

$$
H(\boldsymbol{X} \mid \boldsymbol{Z}) \leq H(c(\boldsymbol{S}), \boldsymbol{U} \mid \boldsymbol{Z})=H(c(\boldsymbol{S}) \mid \boldsymbol{Z})+H(\boldsymbol{U} \mid \boldsymbol{Z}, c(\boldsymbol{S}))
$$

where the term $H(\boldsymbol{U} \mid \boldsymbol{Z}, c(\boldsymbol{S}))$ essentially vanishes since, as mentioned above, every bin forms a channel sub-code that is reliably decodable by the eavesdropper. On the other hand,

$$
H(\boldsymbol{X} \mid \boldsymbol{Z})=H(\boldsymbol{X})-I(\boldsymbol{X} ; \boldsymbol{Z})
$$

thus the equivocation achieved is:

$$
H(\boldsymbol{S} \mid \boldsymbol{Z}) \geq H(c(\boldsymbol{S}) \mid \boldsymbol{Z}) \sim H(\boldsymbol{X})-I(\boldsymbol{X} ; \boldsymbol{Z})
$$

where the first term in the r.h.s. is essentially $n\left[H(S) / \lambda+I\left(X^{*} ; Z^{*}\right)\right]$ and the second term, which is a mutual information induced by a code above capacity, can be evaluated using our above results, provided that the channel code is randomly selected from an ensemble that satisfies our assumptions. For example, if the codewords are chosen i.i.d. according to the distribution of $X^{*}$, then $I(\boldsymbol{X} ; \boldsymbol{Z})$ is approximately $n I\left(X^{*} ; Z^{*}\right)$, and then full secrecy is achieved as $H(\boldsymbol{S} \mid \boldsymbol{Z}) / N$ is essentially equal to $H(S)$. Nonetheless, since the rate of the code $\left[H(S) / \lambda+I\left(X^{*} ; Z^{*}\right)\right.$ is less than $I\left(X^{*} ; Y^{*}\right)$, the legtimate decoder can still decode reliably. Out results can be used also to assess the secrecy achieved by random varlaibles other than i.i.d. according to $X^{*}$, while ensuring that the good decode can still decode reliably.

\section{$6 \quad$ Extension to Multiuser Settings}

The above ideas can be extended in a natural manner to multiuser communication situations, and in this section, we demonstrate this for the multiple access channel (MAC), where the underlying principle is again thermal equilibrium between the subsystems pertaining to the different users and 
that of the channel. As before, our focus is on the regime where reliable communication cannot hold (the paramegnetic phase).

As an example, consider a randomly selected joint source-channel code for a MAC with two users, in the following setting. We are given two independent sources, $S_{1}, S_{2}, \ldots$ and $T_{1}, T_{2}, \ldots$ governed by probability distributions $P_{S}(\cdot)$ and $P_{T}(\cdot)$, which are proportional to $\exp \left\{-\beta \mathcal{E}_{S}(\cdot)\right\}$ and to $\exp \left\{-\beta \mathcal{E}_{T}(\cdot)\right\}$, with partition functions $Z_{S}(\beta)$ and $Z_{T}(\beta)$, respectively. Each $N$-vector of the first source $\boldsymbol{s}=\left(s_{1}, \ldots, s_{N}\right) \in \mathcal{S}^{N}$ is encoded into a channel input vector $\boldsymbol{x}_{S}(\boldsymbol{s}) \in \mathcal{X}_{S}^{n}$ and each $N$-vector of the second source $\boldsymbol{t}=\left(t_{1}, \ldots, t_{N}\right) \in \mathcal{T}^{N}$ is encoded into a channel input vector $\boldsymbol{x}_{T}(\boldsymbol{t}) \in \mathcal{X}_{T}^{n}$. Both codebooks are selected independently, where each codevector of the first code is chosen independently according to distribution $M_{S}$ and each codevector of the second codebook is selected independently according to distribution $M_{T}$. Both codewords are fed into a memoryless MAC $W\left(\boldsymbol{y} \mid \boldsymbol{x}_{S}, \boldsymbol{x}_{T}\right)$, which is proportional to $\exp \left\{-\beta \mathcal{E}_{C}\left(\boldsymbol{x}_{S}, \boldsymbol{x}_{T}, \boldsymbol{y}\right)\right\}$. If we wish to estimate the mutual information $\boldsymbol{E} I(\boldsymbol{S}, \boldsymbol{T} ; \boldsymbol{Y})$ induced by the code, this is quite a trivial extension of the former derivation. But what about $\boldsymbol{E} I(\boldsymbol{S} ; \boldsymbol{Y})$ ?

Here, it will be more convenient to adopt the alternative derivation of eq. (14). Considering the partition function

$$
Z(\beta \mid \boldsymbol{y})=\sum_{\boldsymbol{s}, \boldsymbol{t}} \exp \left\{-\beta\left[\mathcal{E}_{S}(\boldsymbol{s})+\mathcal{E}_{T}(\boldsymbol{t})+\mathcal{E}_{C}\left(\boldsymbol{x}_{S}(\boldsymbol{s}), \boldsymbol{x}_{T}(\boldsymbol{t}), \boldsymbol{y}\right)\right]\right\}
$$

let $\epsilon_{S}^{*}, \epsilon_{T}^{*}$, and $\epsilon_{C}^{*}$ denote the dominant energies allocated to the source $S$, the source $T$, and the MAC, respectively. Also, for a typical randomly chosen codeword $\boldsymbol{x}_{S}(\boldsymbol{s})$ of the source message $\boldsymbol{s}$ actually transmitted, let us define $e^{n \phi_{n, \delta}\left(\epsilon \mid \boldsymbol{x}_{S}(\boldsymbol{s}), \boldsymbol{y}\right)}$ as the probability (under $M_{T}$ ) that $\mathcal{E}_{c}\left(\boldsymbol{x}_{S}(\boldsymbol{s}), \boldsymbol{X}_{T}, \boldsymbol{y}\right)$ is between $n(\epsilon-\delta / 2)$ and $n(\epsilon+\delta / 2)$, for given $\boldsymbol{x}_{S}(\boldsymbol{s})$ and $\boldsymbol{y}$, and assume that as $n \rightarrow \infty$ and then $\delta \rightarrow 0, \phi_{n, \delta}\left(\epsilon \mid \boldsymbol{x}_{S}(\boldsymbol{s}), \boldsymbol{y}\right)$ tends uniformly almost surely to a certain function which will be denoted by $\phi(\epsilon \mid S)$. Now,

$$
\begin{aligned}
\lim _{N \rightarrow \infty} \frac{\boldsymbol{E} I(\boldsymbol{S} ; \boldsymbol{Y})}{N}= & \lim _{N \rightarrow \infty} \frac{1}{N} \boldsymbol{E}\{\ln P(\boldsymbol{Y} \mid \boldsymbol{S})\}-\lim _{N \rightarrow \infty} \frac{1}{N} \boldsymbol{E}\{\ln P(\boldsymbol{Y})\} \\
= & \lim _{N \rightarrow \infty} \frac{1}{N} \boldsymbol{E}\left\{\ln \left[\frac{1}{Z_{T}(\beta)} \sum_{\boldsymbol{t}} \exp \left\{-\beta\left[\mathcal{E}_{T}(\boldsymbol{t})+\mathcal{E}_{C}\left(\boldsymbol{X}_{S}(\boldsymbol{S}), \boldsymbol{X}_{T}(\boldsymbol{t}), \boldsymbol{Y}\right)\right]\right\}\right]\right\}- \\
& \lim _{N \rightarrow \infty} \frac{1}{N} \boldsymbol{E}\left\{\operatorname { l n } \left[\frac { 1 } { Z _ { S } ( \beta ) Z _ { T } ( \beta ) } \sum _ { \boldsymbol { s } , \boldsymbol { t } } \operatorname { e x p } \left\{-\beta\left[\mathcal{E}_{S}(\boldsymbol{s})+\right.\right.\right.\right.
\end{aligned}
$$




$$
\begin{aligned}
& \left.\left.\left.\left.\mathcal{E}_{T}(\boldsymbol{t})+\mathcal{E}_{C}\left(\boldsymbol{X}_{S}(\boldsymbol{s}), \boldsymbol{X}_{T}(\boldsymbol{t}), \boldsymbol{Y}\right)\right]\right\}\right]\right\} \\
= & \psi_{S}(\beta)+\Sigma_{T}\left(\epsilon_{T}^{*}\right)+\lambda \phi\left(\epsilon_{C}^{*} \mid S\right)-\beta\left(\epsilon_{C}^{*}+\epsilon_{T}^{*}\right)-\Sigma_{T}\left(\epsilon_{T}^{*}\right)- \\
& \Sigma_{S}\left(\epsilon_{S}^{*}\right)-\lambda \phi\left(\epsilon_{C}^{*} \mid S\right)+\beta\left(\epsilon_{S}^{*}+\epsilon_{T}^{*}+\epsilon_{C}^{*}\right) \\
= & \lambda\left[\phi\left(\epsilon_{C}^{*} \mid S\right)-\phi\left(\epsilon_{C}^{*}\right)\right]
\end{aligned}
$$

The last line of the above chain of equalities can be intuitively explained as follows: The term $-\lambda \phi\left(\epsilon_{C}^{*}\right)$ stands for $\lim _{N \rightarrow \infty} \boldsymbol{E} I(\boldsymbol{S}, \boldsymbol{T} ; \boldsymbol{Y}) / N$, because of the same reasoning as before (if we look at the pair $(\boldsymbol{S}, \boldsymbol{T})$ as one entity). The term $\lambda \phi\left(\epsilon_{C}^{*} \mid S\right)$ corresponds to the conditional mutual information rate $\lim _{N \rightarrow \infty} \boldsymbol{E} I(\boldsymbol{T} ; \boldsymbol{Y} \mid \boldsymbol{S}) / N$ since the true $\boldsymbol{S}$ is given and only the random codeword of $\boldsymbol{T}$ is selected. Thus, by the chain rule of the mutual information, the difference gives the mutual information rate between $\boldsymbol{S}$ and $\boldsymbol{Y}$.

Example 2. Consider the binary modulo-2 additive MAC, $Y=X_{S} \oplus X_{T} \oplus V$, where all variables take on values in $\{0,1\}, \oplus$ denotes addition modulo 2 (XOR), and $V$ is Bernoulli with parameter $p=\operatorname{Pr}\{V=1\}$, independent of $X_{T}$ and $X_{S}$. Similarly as in Example 1, let the codebooks of the two users be generated by i.i.d. distributions with parameters $m_{S}$ and $m_{T}$, respectively. Now, as before, $\epsilon_{C}^{*}=p$ and the probability that $\boldsymbol{X}_{S} \oplus \boldsymbol{X}_{T}$, whose components are Bernoulli $\left(m_{S} * m_{T}\right)$, would fall within distance $n p$ from a typical $\boldsymbol{y}$, whose components are $\operatorname{Bernoulli}\left(m_{S} * m_{T} * p\right)$, is exponentially $e^{n\left[h_{2}(p)-h_{2}\left(m_{S} * m_{T} * p\right)\right]}$, thus $\phi(p)=h_{2}\left(m_{S} * m_{T} * p\right)-h_{2}(p)$. On the other hand, the probability of the same event conditioned on $\boldsymbol{x}_{S}$, is the probability that $\boldsymbol{X}_{T}$ would fall within distance $n p$ from $\boldsymbol{y} \oplus \boldsymbol{x}_{S}=\boldsymbol{x}_{T} \oplus \boldsymbol{v}$ (which has Bernoulli $\left(m_{T} * p\right)$ components), and thus is of the exponential order of $e^{n \phi(p \mid S)}=e^{n\left[h_{2}(p)-h_{2}\left(m_{T} * p\right)\right]}$. It follows then that

$$
\lim _{N \rightarrow \infty} \frac{\boldsymbol{E} I(\boldsymbol{S} ; \boldsymbol{Y})}{N}=\lambda\left[h_{2}\left(m_{S} * m_{T} * p\right)-h_{2}\left(m_{T} * p\right)\right]
$$

In the special case where $m_{T}=1 / 2$, we get $\lim _{N \rightarrow \infty} \frac{I(\boldsymbol{S} ; \boldsymbol{Y})}{N}=0$ regardless of $m_{S}$, in agreement with intuition, as $\boldsymbol{X}_{T}$ behaves like Bernoulli(1/2) noise in the paramagnetic regime.

\section{References}

[1] S. Arimoto, "On the converse to the coding theorem for discrete memoryless channels," IEEE Trans. Inform. Theory, vol. IT-19, no. 5, pp. 357-359, May 1973. 
[2] I. Csiszár and J. Körner, Information Theory: Coding Theorems for Discrete Memoryless Systems. New York: Academic, 1981.

[3] B. Derrida, "Random-energy model: limit of a family of disordered models," Phys. Rev. Lett., vol. 45, no. 2, pp. 79-82, July 1980.

[4] B. Derrida, "The random energy model," Physics Reports (Review Section of Physics Letters), vol. 67 , no. 1, pp. 29-35, 1980.

[5] B. Derrida, "Random-energy model: an exactly solvable model for disordered systems," Phys. Rev. B, vol. 24, no. 5, pp. 2613-2626, September 1981.

[6] G. Dueck and J. Körner, "Reliability function of a discrete memoryless channel at rates above capacity," IEEE Trans. Inform. Theory, vol. IT-25, no. 1, pp. 82-85, January 1979.

[7] G. D. Forney, Jr., "Exponential error bounds for erasure, list, and decision feeedback schemes," IEEE Trans. Inform. Theory, vol. IT-14, no. 2, pp. 206-220, March 1968.

[8] R. G. Gallager, Information Theory and Reliable Communication, J. Wiley \& Sons, 1968.

[9] D. Guo, S. Shamai, and S. Verdú, "Mutual information and minimum mean-square error in Gaussian channels," IEEE Trans. Inform. Theory, vol. 51, no. 4, pp. 1261-1282, April 2005.

[10] C. Kittel, Elementary statistical physics, John Wiley \& Sons, 1958.

[11] N. Merhav, "The random energy model in a magnetic field and joint source-channel coding," Physica A: Statistical Mechanics and Its Applications, vol. 387, issue 22, pp. 5662-5674, September 15, 2008.

doi:10.1016/j.physa.2008.05.040

[12] M. Mézard and A. Montanari, Information, Physics and Computation, draft, November 2007 [http://www.stanford.edu/ montanar/BOOK/book.html].

[13] P. Ruján, "Finite temperature error-correcting codes," Phys. Rev. Let., vol. 70, no. 19, pp. 2968-2971, May 1993.

[14] A. D. Wyner, "The wire-tap channel," Bell System Technical Journal, vol. 54, no. 8, pp. 13551387, October 1975. 